\title{
Crosswind Shear Gradient Affect on Wake Vortices
}

\author{
Fred H. Proctor* and Nash'at N. Ahmad ${ }^{\dagger}$ \\ NASA Langley Research Center, Hampton, VA 23681-2199 USA
}

\begin{abstract}
Parametric simulations with a Large Eddy Simulation (LES) model are used to explore the influence of crosswind shear on aircraft wake vortices. Previous studies based on field measurements, laboratory experiments, as well as LES, have shown that the vertical gradient of crosswind shear, i.e. the second vertical derivative of the environmental crosswind $\left(\partial^{2} u_{\mathrm{c}} / \partial z^{2}\right)$, can influence wake vortex transport. The presence of nonlinear vertical shear of the crosswind velocity can reduce the descent rate, causing a wake vortex pair to tilt and change in its lateral separation. The LES parametric studies confirm that the vertical gradient of crosswind shear does influence vortex trajectories. The parametric results also show that vortex decay from the effects of shear are complex since the crosswind shear $\left(\partial u_{\mathrm{c}} / \partial z\right)$, along with the vertical gradient of crosswind shear, can affect whether the lateral separation between wake vortices is increased or decreased. If the separation is decreased, the vortex linking time is decreased, and a more rapid decay of wake vortex circulation occurs. If the separation is increased, the time to link is increased, and at least one of the vortices of the vortex pair may have a longer life time than in the case without shear. In some cases, the wake vortices may never link.
\end{abstract}

\section{Nomenclature}

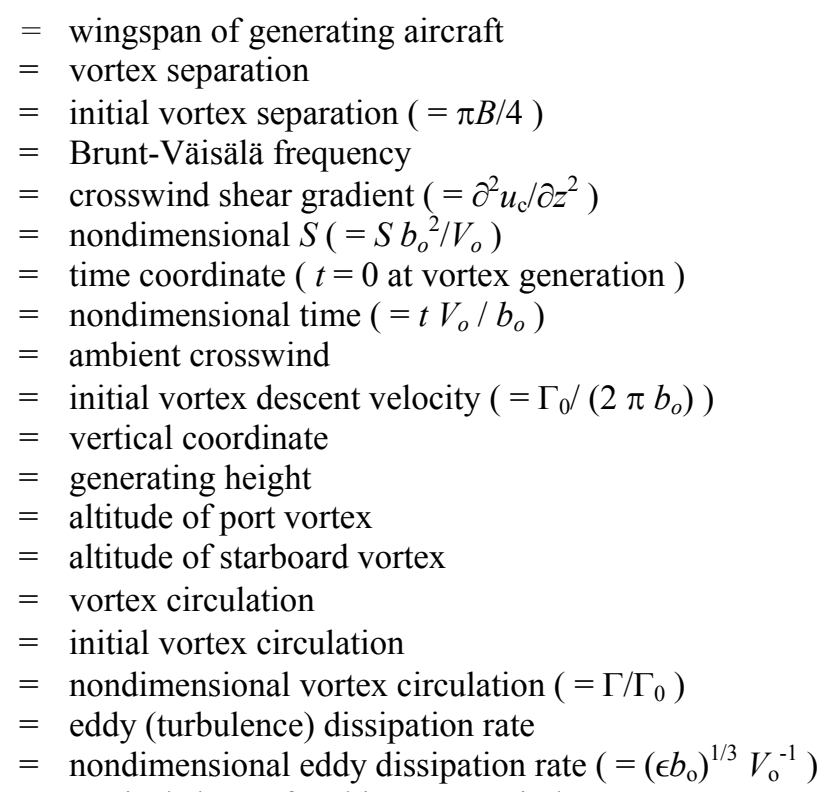

\section{Introduction}

Away from the influence of the ground, a wake-vortex pair typically descends due to its mutual induction while being transported horizontally with the environmental wind. The descent speed of the vortex pair decreases with

\footnotetext{
* Aerospace Technologist, Crew Systems \& Aviation Operations Branch, Mail Stop 152, AIAA Senior Member.

${ }^{\dagger}$ Research Aerospace Engineer, Crew Systems \& Aviation Operations Branch, Mail Stop 152, AIAA Senior Member.
} 
time as the circulation weakens or as the vortices become further apart. However, the presence of an environment with a vertical gradient in crosswind shear can alter the transport and decay of aircraft wake vortices. From field studies, ${ }^{1,2}$ laboratory experiments, ${ }^{3,4}$ and numerical simulations, ${ }^{5,6,7,8,9,10,11}$ the vertical gradient of crosswind shear can preferentially reduce the descent speed of vortices, causing them to tilt, increase separation, and sometimes even to rise upward. Previous numerical investigations with the Terminal Area Simulation System (TASS) have shown this effect to be related to the second derivative of ambient crosswind, rather than the vertical shear of the crosswind. ${ }^{8}$ This was also confirmed in the laboratory experiments conducted by Delisi and Robins. ${ }^{4}$

The schematic in Figure 1 illustrates how the tilt of a vortex pair is affected by the sign of the second derivative of the ambient crosswind. When the second derivative of the crosswind shear is negative, the counter-clockwise rotating vortex (depicted by a plus sign) rises relative to the position of clockwise rotating vortex (depicted by minus sign). The opposite occurs if the sign of the crosswind shear gradient is reversed. In this example, if the crosswind also increases with altitude, then the slower descending vortex would translate more quickly in the lateral direction than the faster descending vortex. This would cause an increasing vertical separation between the counter-rotating vortices and change the characteristic length scales that are based on the initial vortex circulation. The magnitude of descent velocity for each of the vortices would further decrease as the vortices move farther apart. In an opposite fashion, if the upwind vortex were to descend at a slower rate than the downwind as in the case of a positive crosswind shear gradient, the separation of the vortices would decrease.
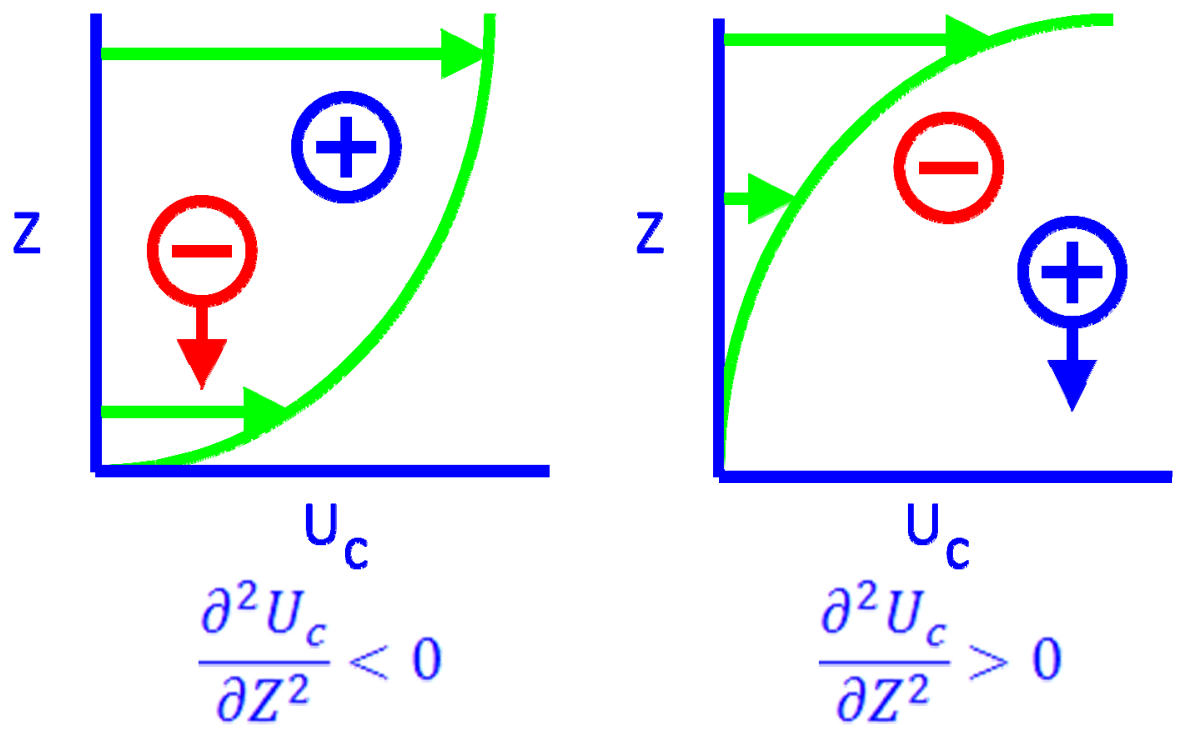

Figure 1. Schematic of crosswind shear effect on a wake vortex pair. Green curve represents vertical profile of crosswind when gradient of crosswind shear is either negative or positive. Assuming a right-handed coordinate system directed along the aircraft path, the port vortex is represented by the red circle, and the starboard by the blue circle.

Although many of the effects of crosswind shear gradient on vortex transport are understood, little is known about how shear affects circulation decay. The observation of unequal vortex decay rates is not unusual during field measurement campaigns; and in some instances, the differential decay has led to the occurrence of solitary vortices that have prolonged lifetimes (e.g., B-757 Case 9 of the Idaho Falls test flights ${ }^{12}$ ).

Fast-time wake vortex prediction models are being advanced for operational applications as well as for use in safety-benefits studies. ${ }^{13}$ Understanding of how crosswind shear affects wake vortex transport and decay is needed in order to improve the accuracy of the fast-time models. Most fast-time models ignore effects from the gradient of crosswind shear and assume both members of a vortex pair descend and decay at the same rate. However, in some instances the shear effects can be very important, since during unfavorable shear conditions wake vortices could linger within the flight corridor and persist over a longer period of time.

In this paper we will use a numerical model to examine the behavior of wake vortices subject to idealized profiles of crosswind. In the following sections the setup and results of the parametric study are described. 


\section{Approach}

The Terminal Area Simulation System (TASS) is a three-dimensional large eddy simulation (LES) model that has been used in the past to study atmospheric and ground influences on wake vortex behavior. The TASS model is described in Refs $[14,15,16]$ and validation studies are described in Refs $[17,18,19]$. The sensitivity of crosswind on vortex descent is examined from two sets of parametric experiments with TASS. Initial conditions are chosen to represent the wake vortices generated by a large category aircraft (B-737) and a heavy category aircraft (B-767). Environmental conditions are set to minimize the effects of turbulence $\left(\epsilon^{*} \approx 0.1\right)$ and stratification $(N=0)$ on vortex decay. Also, the wake vortices are initiated far enough from the ground to minimize any effect the ground surface may have.

Sensitivity of vortex transport and decay is examined over a range of values for the gradient of crosswind shear. The vertical second derivative of ambient crosswind, or gradient of crosswind shear is defined by $S=\partial^{2} u_{\mathrm{c}} / \partial z^{2}$, and is assumed to be uniform within the initial domain. This parameter is nondimensionalized by the initial vortex separation, $b_{o}$, and the initial vortex descent rate, $V_{o}$, as $S^{*}=S b_{o}{ }^{2} / V_{o}$. Experiments with two aircraft types are conducted since one of the aims is to develop a nondimensional relationship that is independent of aircraft type. Values assumed for $S^{*}$ in this study range from 0 to \pm 0.5 . Obviously if $S^{*}$ is a nontrivial constant, the value for $\partial u_{\mathrm{c}} / \partial z$ will change with altitude. Therefore, experiments also are conducted for both $\partial u_{\mathrm{c}} / \partial z>0$, and $\partial u_{\mathrm{c}} / \partial z<0$.

Turbulence for these experiments was grown by surface heating prior to adding the environmental crosswind. Tests were conducted to confirm that the initial crosswind profiles were not modified by the light turbulence during a three minute span equivalent to the lifetime of the wake vortices. The domain size assumed for the simulations was $450 \mathrm{~m} \times 650 \mathrm{~m} \times 455 \mathrm{~m}$ in the longitudinal, lateral, and vertical directions, respectively. The grid size was set to $1.5 \mathrm{~m}$ in the longitudinal direction and $1.25 \mathrm{~m}$ in the lateral and vertical directions. The lateral separation of the initial vortex pair, $b_{o}$, was assumed as $23 \mathrm{~m}$ for the conditions representing the B-737 and $36 \mathrm{~m}$ for the conditions representing the B-767. The initial circulation was assumed to be $250 \mathrm{~m}^{2} \mathrm{~s}^{-1}$ for the B-737 cases and $360 \mathrm{~m}^{2} \mathrm{~s}^{-1}$ for the B-767 cases.

\section{Results}

Results from the parametric simulations confirm that the crosswind shear gradient controls vortex tilt, with the port vortex descending slower for $S^{*}>0$, and the starboard vortex descending more slowly when $S^{*}<0$. This is true irrespective of the sign of the crosswind shear. However, the crosswind shear, $\partial u_{\mathrm{c}} / \partial z$, is important in determining the rate at which the port and starboard vortex spread. For example, if $S^{*}<0$, the starboard vortex will be tilted upward from the port vortex; and if $\partial u_{\mathrm{c}} / \partial z>0$, the starboard vortex will be subject to a relatively stronger crosswind and will increase its lateral separation from the port vortex. Given the same $S^{*}$, but now if $\partial u_{\mathrm{c}} / \partial z<0$, the starboard vortex would be exposed to a weaker crosswind than the port vortex, and a decreasing separation would follow. The TASS results show that the longest lived vortex is determined by the sign of the crosswind shear. If the vorticity associated with the crosswind shear is of opposite sign to the vorticity of the wake vortex, its lifetime will be shorter than the member who has vorticity with same sign as the crosswind shear. For example, if $\partial u_{\mathrm{c}} / \partial z>0$, the port vortex will live longer than the starboard vortex. These general conditions are summarized in Table 1.

Table 1. Effect of crosswind shear on vortex pair.

\begin{tabular}{|ccccc|}
\hline $\begin{array}{c}S^{*} \text { or } \\
\partial^{2} u_{\mathrm{d}} / \partial z^{2}\end{array}$ & $\partial u_{\mathrm{d}} \partial z$ & Tilt & $\begin{array}{c}\text { Change in Lateral } \\
\text { Spread }\end{array}$ & $\begin{array}{c}\text { Longest Lived } \\
\text { Vortex }\end{array}$ \\
\hline$<0$ & $<0$ & $\mathrm{Z}_{\mathrm{s}}>\mathrm{Z}_{\mathrm{p}}$ & decreasing & starboard \\
\hline$<0$ & $>0$ & $\mathrm{Z}_{\mathrm{s}}>\mathrm{Z}_{\mathrm{p}}$ & increasing & port \\
\hline$>0$ & $>0$ & $\mathrm{Z}_{\mathrm{s}}<\mathrm{Z}_{\mathrm{p}}$ & decreasing & port \\
\hline$>0$ & $<0$ & $\mathrm{Z}_{\mathrm{s}}<\mathrm{Z}_{\mathrm{p}}$ & increasing & starboard \\
\hline$=0$ & $\neq 0$ & $\mathrm{Z}_{\mathrm{s}}=\mathrm{Z}_{\mathrm{p}}$ & constant & same \\
\hline
\end{tabular}

The decrease or increase in the lateral separation due to the crosswind derivatives also plays an important role in the wake vortex decay. If the vortex lateral separation increases, then the characteristic length scale changes. For no shear cases this length scale is based on $b_{o}$. However, larger values may be more appropriate when the lateral 
separation increases due to the crosswind shear gradient. An increasing vortex separation also would mean that vortex linking due to Crow instability ${ }^{20}$ can be delayed or suppressed. In the case of decreasing vortex separation (Table 1), vortex linking would occur sooner. The nondimensional times when vortex linking occurred in our parametric study are shown in Tables 2 and 3. For conditions when the lateral spread increases, the linking times increase as well (Table 2). In the stronger shear cases, the separation becomes so great that linking does not occur. On the other hand, if the conditions are such that the lateral separation is decreased (Table 3), linking occurs sooner. It should be noted that $\partial u_{\mathrm{c}} / \partial z$ and $S^{*}$, affect the linking time since both control the rate at which the port and starboard vortices spread with time.

Table 2. Effect of negative $S^{*}$ on nondimensional linking time when $\partial u_{\mathrm{c}} / \partial z>0$.

\begin{tabular}{|c|c|c|}
\hline S* & B-737 & B-767 \\
\hline 0.0 & 3.0 & 3.25 \\
\hline-0.15 & 3.0 & 3.4 \\
\hline-0.2 & 3.25 & 3.5 \\
\hline-0.25 & 3.35 & 3.55 \\
\hline-0.3 & $\infty$ & $\infty$ \\
\hline-0.4 & $\infty$ & $\infty$ \\
\hline
\end{tabular}

Normally, the only environmental parameter thought to be important for predicting Crow linking is the turbulence intensity, i.e., $\epsilon^{*}{ }^{21,22,23}$ Our results show that the presence of crosswind shear gradient can introduce great uncertainty into the prediction by either reducing or increasing the time at which of the vortex linking occurs. The ability to quantify the linking time is important in many wake vortex decay models.

Table 3. Effect of positive $S^{*}$ on nondimensional linking time when $\partial u_{c} / \partial>0$.

\begin{tabular}{|c|c|c|}
\hline S* & B-737 & B-767 \\
\hline 0.0 & 3.0 & 3.25 \\
\hline 0.15 & 2.1 & 2.75 \\
\hline 0.2 & 2.0 & 2.45 \\
\hline 0.25 & 1.9 & 2.39 \\
\hline 0.3 & 1.5 & 2.1 \\
\hline 0.4 & 1.4 & 1.8 \\
\hline
\end{tabular}

The three-dimensional visual representation of the tilting effect due to the gradient of crosswind shear is shown in Figure 2. The vortex pair is spreading due to the crosswind shear gradient and tilting upward toward the starboard vortex.

A visualization of one the cases with decreasing separation and accelerated linking time is shown in Figure 3. At the time of plot, the starboard vortex has nearly dissipated into rings, leaving an intact port vortex.

Trajectory and circulation plots for two contrasting cases are shown in Figures 4 and 5. For the case with $S^{*}=-0.3$, and $\partial u_{\mathrm{c}} / \partial z>0$, the vortices never link and the port vortex has the longest lifetime. Even at three minutes, the port vortex has decayed only to $70 \%$ of its initial value, and remains many times stronger than the vortex in the case with no crosswind. For the case with $S^{*}=0.2$, and $\partial u_{\mathrm{c}} / \partial z>0$, the lateral separation contracts, and Crow linking occurs at $t=27 \mathrm{~s}$. Note that both vortices decay faster than the baseline and the port vortex is slightly more persistent. In both Figure 4 and 5, the vortex trajectories are altered by the crosswind, and the vortices do not descend to the same depth as for the cases with no crosswind. 


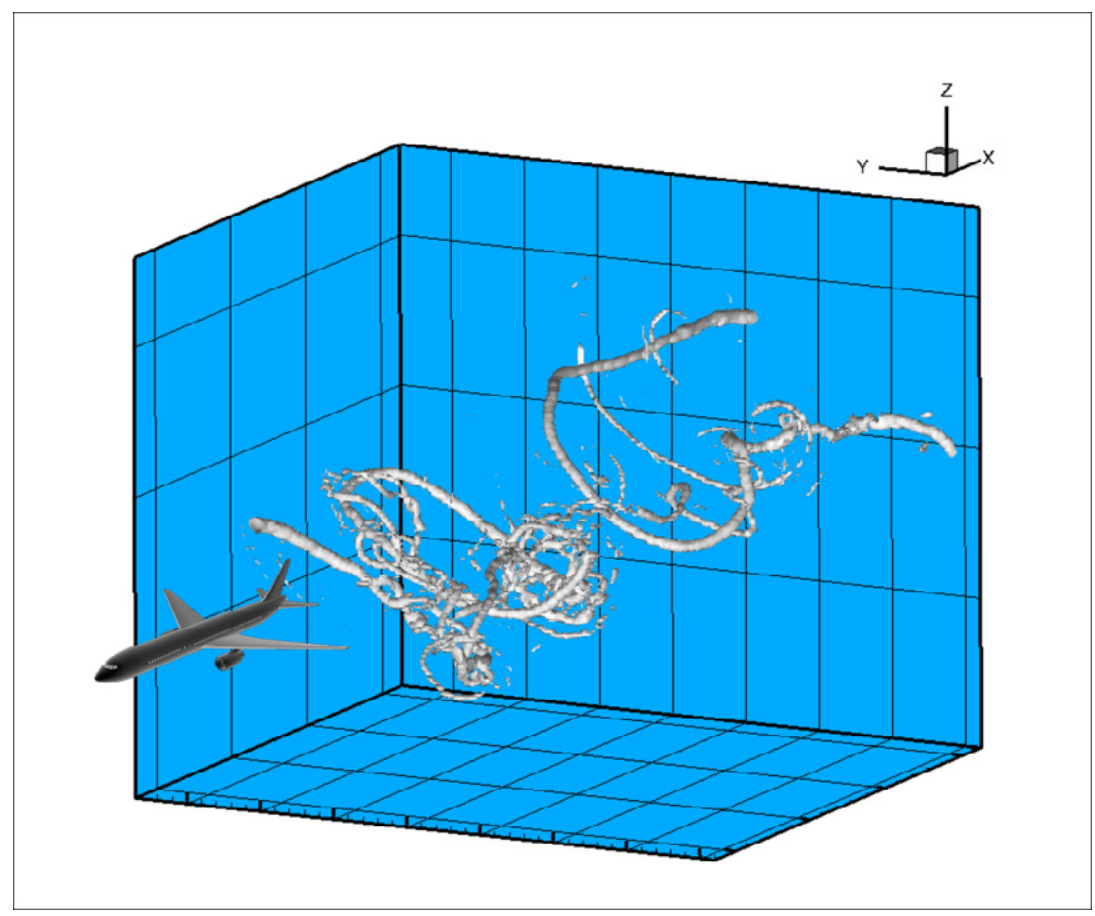

Figure 2. Three-dimensional perspective of the wake vortex pair tilted by shear. Viewed from the port side for the B-767 case with $S^{*}=-0.25$ and $\partial u_{\mathrm{c}} / \partial z>0$, at $t=114 \mathrm{~s}\left(\mathrm{~T}^{*}=5\right)$. Crosswind is to the left.

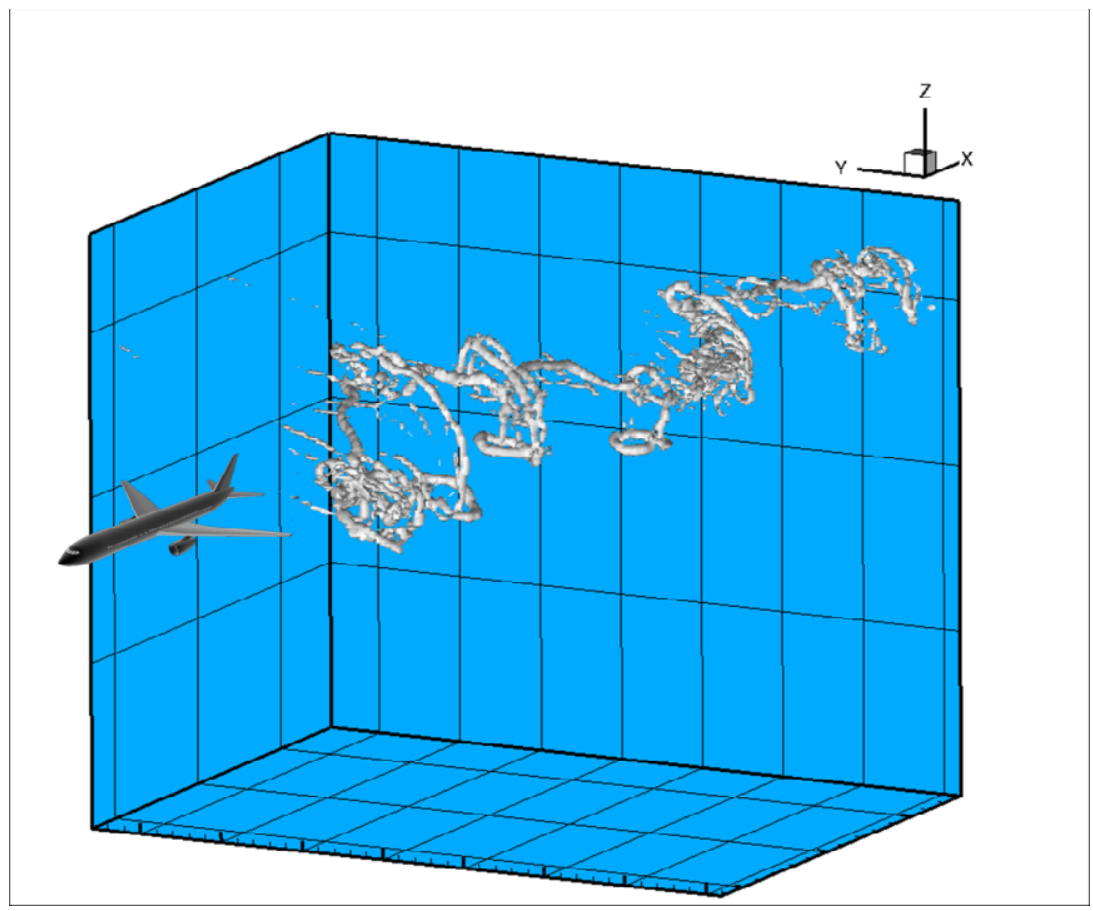

Figure 3. Three-dimensional perspective of the wake with accelerated linking due to shear. Viewed from the port side for the B-737 case with $S^{*}=0.2$ and $\partial u_{\mathrm{c}} / \partial z>0$, at $\mathrm{t}=45 \mathrm{~s}\left(\mathrm{~T}^{*}=3.4\right)$. Crosswind is to the right. 

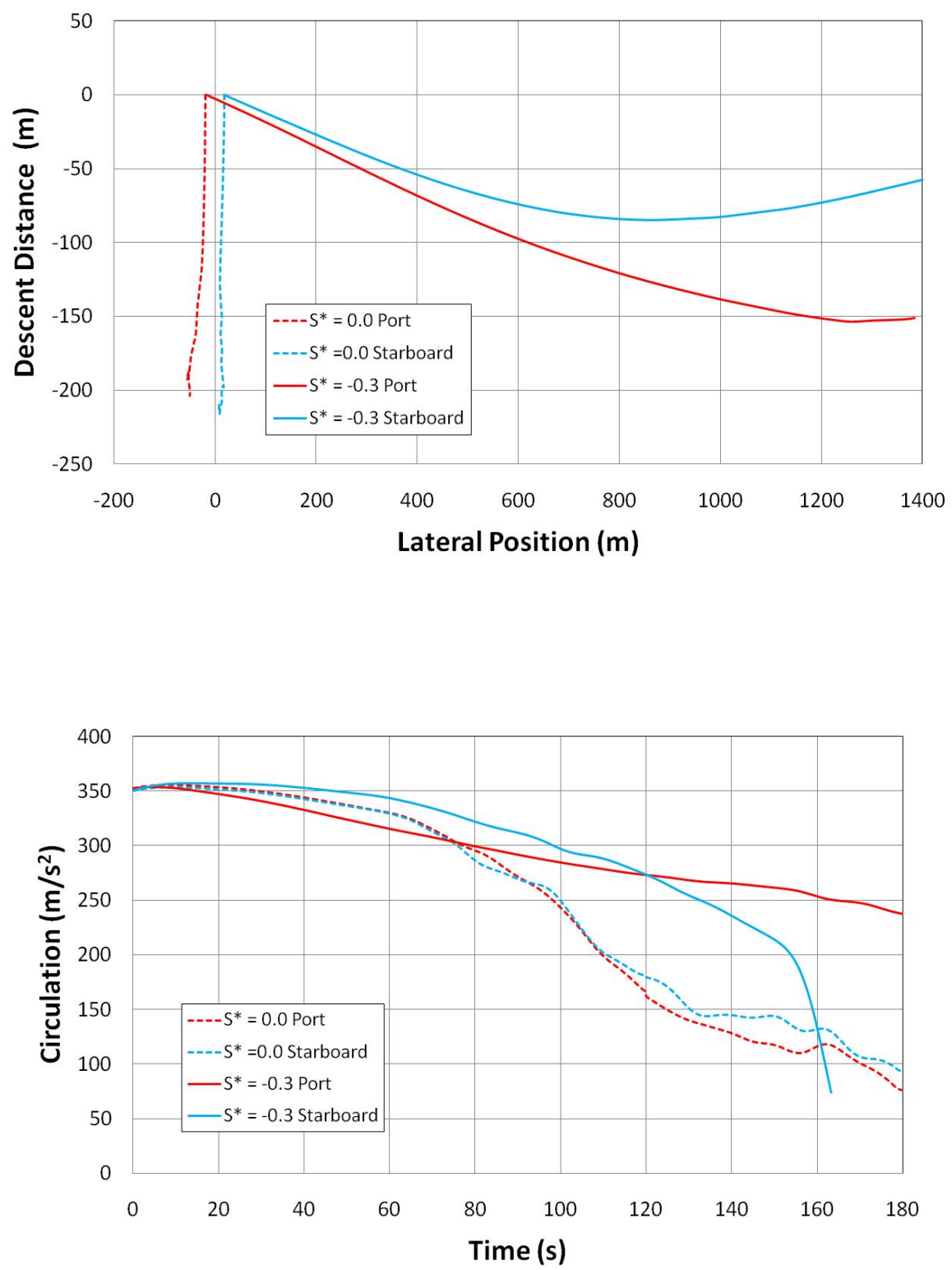

Figure 4. Vortex trajectory (top), and vortex circulation vs. time (bottom) for the B-767 case with $S^{*}=\mathbf{- 0 . 3}$, and $\partial u_{\mathrm{c}} / \partial z>0$. The baseline case for $\mathrm{B}-767$ with no crosswind is shown as dashed line. The port vortex is denoted by red and the starboard vortex by the blue line. 

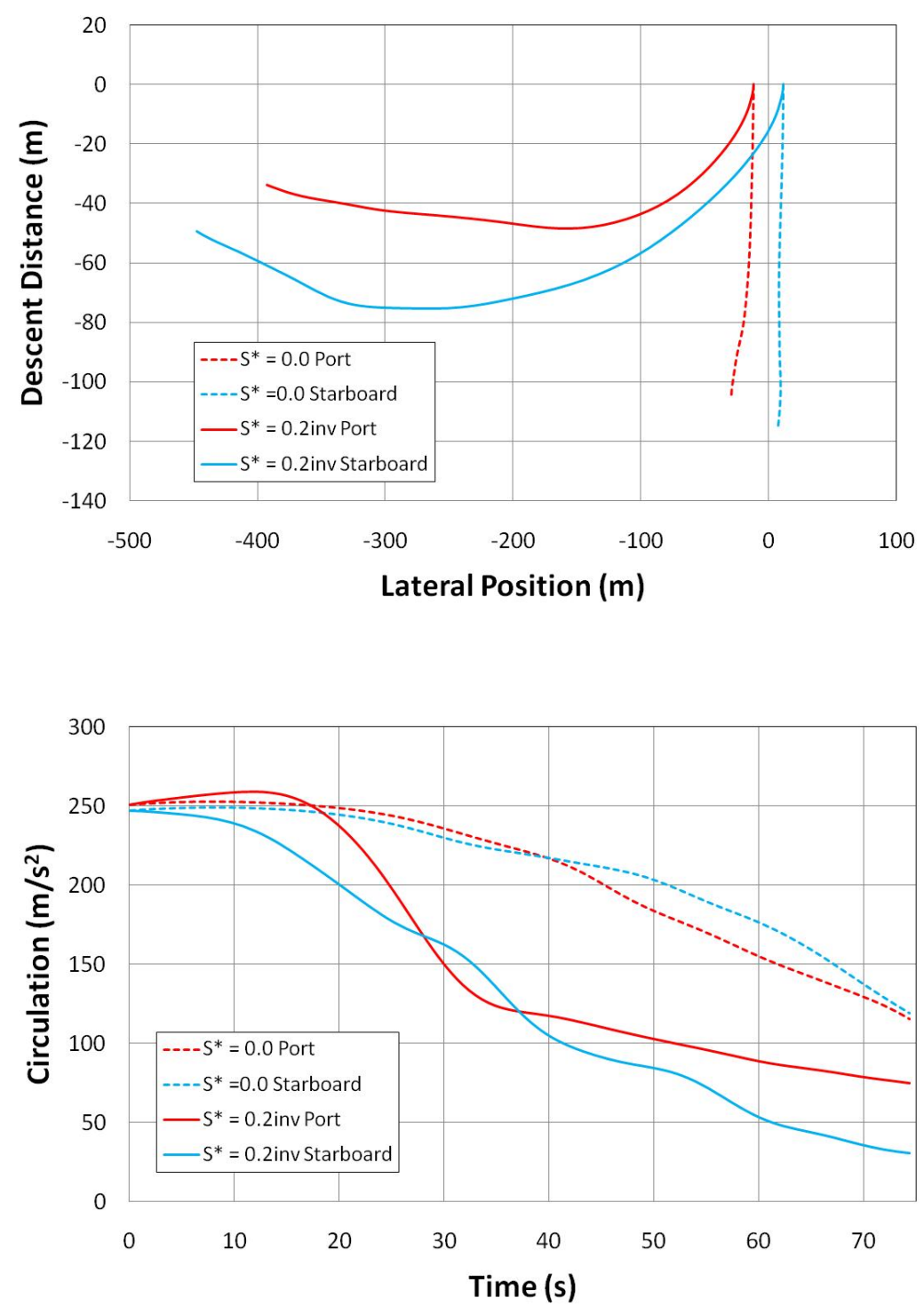

Figure 5. Vortex trajectory (top), and vortex circulation vs. time (bottom) for the B-737 case with $S^{*}=0.2$, $\partial u_{\mathrm{c}} / \partial z>0$ (same case as used in Figure 3). The baseline case for B-737 with no crosswind is shown by the dashed lines. The port vortex is denoted by red and the starboard vortex by blue.

\section{Summary}

A parametric study is conducted with LES to examine the effect of idealized crosswind profiles on the behavior of a wake vortex pair. The crosswind shear gradient causes vortices in a vortex pair to descend at different rates. Once the vortices become tilted, they are transported at different lateral speeds due to the changes in crosswind with altitude (e.g., the higher vortex may be exposed to stronger crosswind). Their separation may increase or decrease depending upon the sign of the crosswind shear. Changes in the vortex separation due to the shear further affect the vortex descent rate, as well as the linking time and the rate of decay. Furthermore, the port and starboard members of the vortex pair may decay at different rates, and the vortex with the same sign of vorticity as the crosswind shear tends to persist longer than the vortex with opposite vorticity to the shear. The results of this study suggest that the 
probabilistic approaches for wake prediction need to account for the greater uncertainty when shear is present, and deterministic approaches that predict time to link and vortex decay need to modify the characteristic scale due to the shear effects.

\section{Acknowledgments}

This work is done in support of the Airspace Systems Program. Acknowledgements are expressed to Fanny Limon Duparcmeur of Metis Technology Solutions and George Switzer of Analytical Services \& Materials. The numerical simulations were conducted using the NASA Pleiades supercomputer cluster.

\section{References}

\footnotetext{
${ }^{1}$ Brashears, M.R., Logan, N.A. and Hallock, J.N., "Effect of Wind Shear and Ground Plane on Aircraft Wake Vortices," J. Aircraft, Vol. 12, 1975, pp. 830-833.

2 Matthews, M.P., Dasey, J.T., Perras, G.H., and Campbell S.D., "Planetary Boundary Layer Measurements for the Understanding of Aircraft Wake Vortex Behavior," 7th Conf. on Aviation Weather Systems, Long Beach, CA, Amer. Meteor. Soc., Paper 5-5, February 1997.

${ }^{3}$ Liu, C. H., and Ting, L., "Interaction of Decaying Trailing Vortices in Spanwise Shear Flow," Computers and Fluids, Vol. 15, No. 1,1987, pp. 77-92.

${ }^{4}$ Delisi, D.P. and Robins, R.E., "Effects of Crosswind Shear on Trailing Vortex Evolution,” January 2006, AIAA $2006-1075$.

${ }^{5}$ Burnham, D. C., "Effect of Ground Wind Shear on Aircraft Trailing Vortices," AIAA Journal, Vol. 10, No. 8, 1972, pp. $1114,1115$.

${ }^{6}$ Robins, R.E., and Delisi, D.P., "Potential Hazard of Aircraft Wake Vortices in Ground Effect with Crosswind," J. Aircraft, Vol. 30, pp. 201-206.

${ }^{7}$ Proctor, F.H., Hinton, D.A., Han, J., Schowalter, D.G., and Lin Y.-L., "Two Dimensional Wake Vortex Simulations in the Atmosphere: Preliminary Sensitivity Studies,” January 1997, AIAA 97-0056.

${ }^{8}$ Proctor, F.H., "The NASA-Langley Wake Vortex Modelling Effort in Support of an Operational Aircraft Spacing System," January 1998, AIAA 98-0589.

${ }^{9}$ Zheng, Z.C. and Baek, K., "Inviscid Interactions Between Wake Vortices and Shear Layers," AIAA Journal, Vol. 36, No. 2, 1999, pp. 477-480.

${ }^{10}$ Proctor, F.H., Hamilton, D.W., and Switzer, G.F. “TASS Driven Algorithms for Wake Prediction,” January 2006, AIAA 2006-1073.

${ }^{11}$ Robins, R.E., and Delisi, D.P., “3-D Numerical Simulations of Trailing Vortex Evolution,” January 2006, AIAA 20061079 .

${ }^{12}$ Garodz, L.J., and Clawson, K.L., "Vortex Wake Characteristics of B757-200 and B767-200 Aircraft Using the Tower FlyBy Technique, Volumes 1 and 2," January 1993, NOAA Tech. Memo. ERL ARL-199.

${ }^{13}$ Proctor, F.H. and Hamilton, D.W., "Evaluation of Fast-Time Wake Vortex Prediction Models,” January 2009, AIAA 20090344.

${ }^{14}$ Proctor, F.H., "The Terminal Area Simulation System, Volume 1: Theoretical Formulation,” April 1987, NASA CR-4046.

${ }^{15}$ Proctor, F.H., "Numerical Simulation of Wake Vortices Measured During the Idaho Falls and Memphis Field Programs,"

$14^{\text {th }}$ AIAA Applied Aerodynamic Conference, Proceedings, Part II, June 1996, AIAA 96-2496, pp. 943-960.

${ }^{16}$ Proctor, F.H., "Interaction of Aircraft Wakes from Laterally Spaced Aircraft," January 2009, AIAA 2009-0343.

${ }^{17}$ Switzer, G.F., "Validation Tests of TASS for Application to 3-D Vortex Simulations," October 1996, NASA CR-4756.

${ }^{18}$ Shen, S., Ding, F., Han, J., Lin, Y.-L., Arya, P., and Proctor, F., "Numerical Modeling of Wake Vortices: Real Case Simulations,' January 1999, AIAA 99-0755.

${ }^{19}$ Ahmad, N.N., and Proctor, F.H., "Simulation of Benchmark Cases with the Terminal Area Simulation System (TASS)," January 2011, AIAA 2011-1005.

${ }^{20}$ Crow, S. C., "Stability Theory for a Pair of Trailing Vortices," AIAA Journal, Vol. 8, No. 12, December 1970, pp. 21722179.

${ }^{21}$ Proctor, F.H., Ahmad, N.N, Switzer, G.F., and Limon Duparcmeur, F.M, “Three-Phased Wake Vortex Decay,” August 2010, AIAA 2010-7991.

${ }^{22}$ Han, J., Lin, Y.-L., Schowalter, D. G., Arya, S. P., and Proctor, F. H., "Large Eddy Simulation of Aircraft Wake Vortices within Homogeneous Turbulence: Crow Instability," AIAA Journal, Vol. 38, February 2000, pp 292-300.

${ }^{23}$ Sarpkaya, T., and Daly, J.J., "Effect of Ambient Turbulence on Trailing Vortices," J. Aircraft, Vol. 24, No. 6, 1987, pp. 399-404.
} 